\title{
Effects of reinforcement duration and ratio size on discrete-trials FR responding*
}

\author{
STEPHEN C. BITGOOD and JOHN R. PLATT \\ McMaster University, Hamilton, Ontario, Canada
}

Two pigeons were given discrete-trials fixed-ratio (FR) training with 2-, 4-, and 8-sec hopper times and ratio sizes of 30 or 60 responses. A final phase consisted of free-responding FR 60. The following results were found: (1) Start speeds and ratio rates were an increasing function of reinforcement duration when the schedule was discrete-trials FR 60, but not when it was FR 30; (2) the FR 60 schedule resulted in a lower level of responding than did the FR 30 schedule; and (3) the positive relationship between performance and reinforcement duration with discrete-trials FR 60 did not persist with free-responding FR 60. These results suggest that discrete-trials procedures may be more sensitive to reinforcement density manipulations than free-responding procedures.

The effects of reinforcement parameters in ratio schedules of reinforcement with pigeons have been studied both by varying the size of the ratio and by varying reinforcement duration (e.g., Powell 1968, 1970). Both of these manipulations have resulted in postreinforcement pauses (latencies of the first response after reinforcement) which are a decreasing function of duration of reinforcement per response. Ratio rates, or response rates excluding the post reinforcement pause, have also tended to show faster responding with longer reinforcement durations and smaller ratio sizes (e.g., Felton \& Lyon, 1966; Powell, 1968, 1969, 1970). However, these ratio-rate results have not been consistent across sessions or across birds. Also, Schwartz (1969) found that response rates in the terminal fixed-ratio (FR) component of a concurrent two-component chain were insensitive to both ratio size and reinforcement duration. Such results have led Powell (1969) to suggest that, while FR postreinforcement pause is sensitive to reinforcement duration and ratio size, response rate after the first response of an FR requirement is not. All of these studies have used a free-responding procedure as opposed to a discrete-trials one in which timeouts (TO) follow each reinforcement. Such a discrete-trials operant procedure has sometimes been treated as analogous to the discrete-trials runway (e.g., Bitgood \& Platt, 1971; Platt, 1971), where running speed has been found to be an increasing function of magnitude of reinforcement (e.g., Capaldi \& Lynch, 1967). The runway data suggest that a discrete-trials modification might result in ratio rates

\footnotetext{
*This research was completed at the University of Iowa and was supported by a National Defense Education Act Fellowship to the first author and by United States Public Health Service Grant MH 15380 (John R. Platt, principal investigator). Requests for reprints should be sent to either author, Department of Psychology, McMaster University, Hamilton, Ontario, Canada.
}

being more sensitive to reinforcement duration, and perhaps to ratio size. Bitgood \& Platt (1971) found evidence consistent with this conjecture in that a smaller ratio (FR 30) resulted in faster ratio rates and start speeds (1/latency of the first response) than a larger ratio (VR 60) when a discrete-trials operant procedure was used. The present study manipulated reinforcement duration and FR size in a discrete-trials operant situation to determine if such a procedure would yield reliable effects on both start speeds and ratio rates. A free-responding condition was also included to assess the effects of reinforcement duration when TOs were absent.

\section{METHOD \\ Subjects}

Two male White Carneaux pigeons, 6 to 8 years of age, were maintained at $80 \%$ of their free-feeding weights. Both birds had served in a previous experiment involving a FR 30 reinforcement schedule.

\section{Apparatus}

Two single-key operant chambers similar to that described by Ferster \& Skinner (1957) were used. Reinforcement was either 2-, 4-, or 8-sec access to Purina Pigeon Checkers in a Lehigh Valley Electronics food hopper. A small pilot lamp served as a houselight and remained on while the key lights or food hopper were activated. Automatic scheduling and storage of data were accomplished simultaneously in both chambers by a Digital Equipment Corporation PDP-8/I computer. Data were recorded by an on-line Teletype.

\section{Procedure}

The birds were initially trained in a manner similar to that described by Bitgood \& Platt (1971). Autoshaping of the keypeck had previously been accomplished according to the procedure described by Brown \& Jenkins (1968). Thirty sessions of training on a discrete-trials FR 30 schedule of reinforcement had been given in a previous experiment. The present study began with training to three values of reinforcement duration in three separate sessions Each session consisted of 30 reinforcements on a FR schedule, with a 10-sec TO after each reinforcement. The TO consisted of a complete blackout of the chamber. For Bird 93, a yellow key light was associated with the 2-sec, a red key light with the 4-sec, and a green key light with the 8-sec reinforcement duration. For Bird 97, red was associated with the 2-sec, green with the 4-sec, and yellow with the 8-sec reinforcement duration. A three-session block with one session of each reinforcement duration constituted a cycle. The three reinforcement durations were randomly ordered within each cycle, with the restriction that each value was used for exactly one session. The cycles at FR 30 were followed by five cycles at FR 60 . The birds were then shifted back to FR 30 for five cycles, followed by nine cycles at FR 60 . Finally, nine cycles of free-responding FR 60 were given.

\section{RESULTS and DISCUSSION}

The latency of each bird's first response on each trial was reciprocalized and averaged for each session to form a start-speed measure. Similarly, the mean number of responses per second, excluding the time for the first 

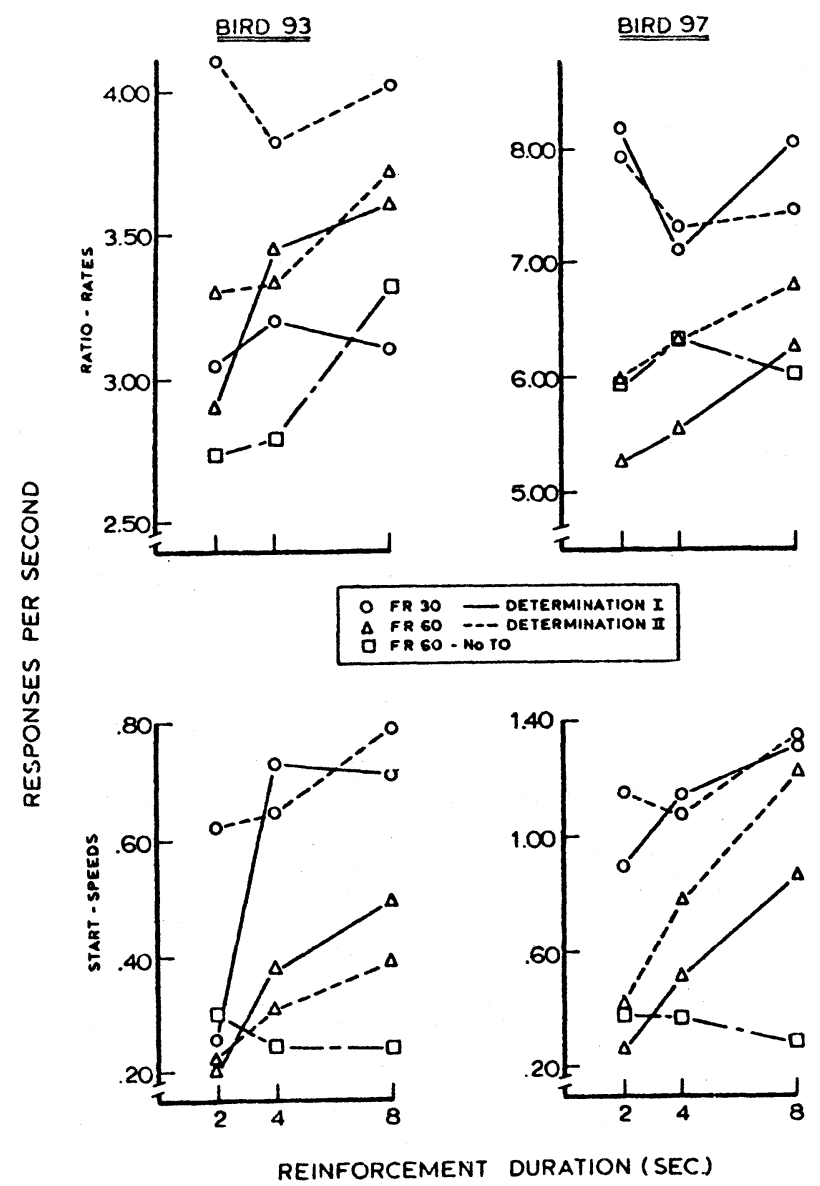

Fig. 1. Mean ratio rates and start speeds for both birds at the three reinforcement duration values. These points are averaged over the last five cycles of each phase.

response, was computed for each bird on each trial to obtain a ratio-rate measure. The results summarized in Fig. 1 represent means over the last five cycies of each phase. The left frames display response measures for Bird 93 and the right frames for Bird 97. Ratio rates are shown on the top and start speeds on the bottom.

As evidenced by Fig. 1, consistent relationships between performance and reinforcement duration only occurred when the ratio size was sufficiently large. Both birds showed a monotonically increasing relationship between ratio rates and reinforcement duration, as well as between start speeds and reinforcement duration, for both phases of discrete-trials FR 60. Such consistency was not found in the FR 30 phases. However, both birds did show slower start-speeds for the 2-sec than for the 4or 8-sec hopper in the FR 30-I phase, suggesting that start speeds were more sensitive to reinforcement duration than were ratio rates. The ratio size and reinforcement duration effects on start speeds were in agreement with previous reports. For example, Powell (1969) found that the effect of reinforcement duration on postreinforcement pause was reduced by decreasing the ratio size. The ratio rate results, on the other hand, do not agree with previous findings in which no consistent relationships between ratio rate and reinforcement duration have been found.

In the final phase of the experiment, free-responding FR 60, an increasing relation between performance and reinforcement duration was obtained only for the ratio rates of Bird 93. The start speeds showed no such relationship, and Bird 97 showed no differential performance for either measure. The lack of differential responding appears to be related to the change from discrete-trials to free-responding procedures, since all other experimental conditions were unchanged. A return to discrete-trials FR 60 would have been more convincing to rule out disruption due to generalization decrement caused by a change in the experimental procedures. However, no improvement in postshift performance was observed over nine cycles (27 sessions) of training. Thus, it appears that generalization decrement is an unlikely explanation for this result.

Increasing the size of the ratio had another effect in addition to increasing sensitivity to reinforcement duration. Slower ratio rates and start speeds were obtained for the FR 60 schedule than for the FR 30 schedule, with the exception of the FR 30-I phase ratio rates of Bird 93. This exception may have been due to nonasymptotic performance in this initial phase. As with reinforcement duration findings for the start speeds, the ratio-size results were consistent with previous reports (e.g., Powell, 1970). However, the usual ratio-rate finding has been an inconsistent relationship between ratio rates and ratio size. Again, the discrete-trials procedure of the present study was probably responsible for this discrepancy. The present results are consistent with those of Bitgood \& Platt (1971), who found a substantial ratio-rate difference between FR 30 and VR 60 with a discrete-trials procedure.

Why should the discrete-trials situation have been more sensitive to reinforcement duration than the free-operant one? The present results do not provide a satisfactory answer to this question; however, at least two accounts seem unlikely. First is the possibility that TO reduces inhibitory aftereffects of reinforcement. For example, the aftereffects of eating, or demotivation, might tend to offset performance increments due to reinforcement duration more in a free-operant situation than in a discrete-trials one. This explanation is not entirely satisfactory in the present case for two reasons. Demotivation would be expected to mask reinforcement duration effects on start speeds more than those on ratio rates, while it has usually been found that start speeds are more sensitive to reinforcement duration than are ratio rates. In addition, we have looked for a demotivation type of effect with pigeons in several experiments where such effect would be expected, but have never found one.

Another account may be proposed for the finding that reinforcement duration effects for ratio rates were obtained with discrete-trials procedures but not with 
free-responding procedures. It may be pointed out that the TO reduced overall reinforcement rate. In the present study, both increasing the ratio size and decreasing the reinforcement duration involved reducing the amount of reinforcement per unit of time. Any manipulation which reduces the overall amount of reinforcement per unit of time may produce similar results. Such an interpretation implies that performance would be faster when the reinforcement rate is increased by removing the TO. However, Fig. 1 reveals that in the FR 60/no-TO phase, a general decrease in start speeds and ratio rates was observed rather than the increase predicted by the reinforcement rate interpretation.

While other explanations are possible for the finding that a discrete-trials procedure was more sensitive to reinforcement duration than a free-responding one, there are little data upon which to make an objective choice. More research is needed to test some of the possible alternative explanations.

\section{REFERENCES}

Bitgood, S. C., \& Platt, J. R. A discrete-trials PREE in an operant situation. Psychonomic Science, 1971, 23, 17-19.

Brown, P. L., \& Jenkins, H. M. A utoshaping of the pigeon's key-peck. Journal of the Experimental Analysis of Behavior, $1968,11,1-8$.

Capaldi, E. J., \& Lynch, D. Repeated shifts in reward magnitude: Evidence in favor of an associational and absolute (contextual) interpretation. Journal of Experimental Psychology, 1967, 75, 226-235.

Felton, M., \& Lyon, D. O. The post-reinforcement pause. Journal of the Experimental Analysis of Behavior, 1966, 9 , 131-134.

Ferster, C. B., \& Skinner, B. F. Schedules of reinforcement. New York: Appleton-Century-Crofts, 1957.

Platt, J. R. Discrete-trials and their relation to free-behavior situations. In J. T. Spence \& H. H. Kendler (Eds.), Essays in neobehaviorism: $A$ memorial to Kenneth $W$. Spence. New York: Appleton-Century-Crofts, 1971.

Powell, $R$. W. The effect of small sequential changes in fixed-ratio size upon the post-reinforcement pause. Journal of the Experimental Analysis of Behavior, 1968, 11, 589-593.

Powell, $R$. W. The effect of reinforcement magnitude upon responding under fixed-ratio schedules. Journal of the Experimental Analysis of Behavior, 1969, 12, 605-608.

Powell, $R$. W. Probability of reinforcement and fixed-ratio responding: A model. Psychological Record, 1970, 20, 73-80.

Schwartz, B. Effects of reinforcement magnitude of pigeons preference for different fixed-ratio schedules of reinforcement. Journal of the Experimental Analysis of Behavior, 1969, 12, 253-259.

Senkowski, P. C. Temporal course of motivational after-effects of reward and nonreward. Unpublished Doctoral Dissertation, University of Iowa, 1970.

Staddon, J. E. R. Temporal effects of reinforcement: A negative "frustration" effect. Learning \& Motivation, 1970, 1 , 227-247.

(Received for publication March 21, 1973.)

\section{Negative contrast in goldfish (Carassius auratus)*}

\section{THERESE L. COCHRANE, STANLEY R. SCOBIE and DANIEL FALLON \\ State University of New York at Binghamton \\ Binghamton, N.Y. 13901}

Goldfish in a successive differential discrimination displayed strong negative contrast. A successive nondifferential shift in reward magnitude did not produce clear negative contrast, however. Response speed changed appropriately when reward magnitudes were changed. There was no indication of positive contrast in either condition.

Much work on contrasted conditions of reward has been done with rats as Ss, but work with goldfish (Bitterman, 1969; Lowes \& Bitterman, 1967; Mackintosh, 1971; Raymond, Aderman, \& Wolach, 1972) has failed to

*This is a preliminary report of aspects of research to be subsequently reported in greater detail. The research was supported by MH 19961 from the National Institute of Mental Health. demonstrate either positive or negative contrast. These "negative" findings were partly responsible for Bitterman's (1969) description of fish as animals primarily responsive to laws governing simple stimulus-response bonds. The demonstration of contrast in goldfish would seem to imply behavior which is not readily accounted for in terms of simple stimulus-response bonds, and would be of substantial theoretical interest.

One criterion we can use to classify successive "contrast effects" differentiates situations in which the organism has the opportunity to compare different reward magnitudes directly, within a daily trial sequence, from situations in which reward magnitudes are experienced in separate successive blocks in time (Dunham, 1968). The first case, successive differential contrast, typically involves intermixed trials, signaled by different stimuli and resulting in different magnitudes of reward. Rats in such a situation usually display negative contrast, responding more slowly on small reward trials than do animals given the same small reward presented alone, 